\begin{tabular}{|c|c|c|}
\hline Case Reports in & \multicolumn{2}{|c|}{ Case Rep Gastroenterol 2020;14:103-109 } \\
\hline Gastroenterology & $\begin{array}{l}\text { DOI: } 10.1159 / 000506186 \\
\text { Published online: February } 25,2020\end{array}$ & $\begin{array}{l}\text { (c) } 2020 \text { The Author(s) } \\
\text { Published by S. Karger AG, Basel } \\
\text { www.karger.com/crg }\end{array}$ \\
\hline & $\begin{array}{l}\text { This article is licensed under the } \\
\text { International License (CC BY-NC) } \\
\text { Usage and distribution for commercia }\end{array}$ & $\begin{array}{l}\text { nons Attribution-NonCommercial } 4.0 \\
\text { ger.com/Services/OpenAccessLicense). } \\
\text { uires written permission. }\end{array}$ \\
\hline
\end{tabular}

\title{
Cytomegalovirus Enterocolitis in a Patient with Refractory Immune-Related Colitis
}

\author{
Yoki Furuta $^{a}$ Hideaki Miyamoto $^{a}$ Hideaki Naoe $^{a}$ Miki Shimoda $^{a}$ \\ Yukari Hinokuma $^{a}$ Tomohiro Miyamura $^{\mathrm{b}}$ Azusa Miyashita $^{\mathrm{b}}$ \\ Satoshi Fukushima ${ }^{b}$ Motohiko Tanaka $^{a}$ Yutaka Sasaki ${ }^{a, c}$ \\ aDepartment of Gastroenterology and Hepatology, Graduate School of Medical Sciences, \\ Kumamoto University, Kumamoto, Japan; ${ }^{b}$ Department of Dermatology and Plastic \\ Surgery, Kumamoto University, Kumamoto, Japan; 'Kaizuka City Hospital, Kaizuka, Japan
}

\section{Keywords}

Colitis · Cytomegalovirus · Immune-related adverse event · Immunotherapy

\section{Abstract}

Although most immune-related adverse events (irAEs) secondary to immune checkpoint inhibitors can be managed with immunosuppressive therapies; they can induce reactivation of infectious diseases, including cytomegalovirus (CMV). Here, we show a case of CMV enterocolitis during steroid therapy for an irAE. A 77-year-old man with unresectable malignant melanoma was treated with ipilimumab. He suffered from immune-related colitis (irColitis) and was treated with methylprednisolone. Although corticosteroids initially improved his symptoms, CMV reactivation occurred and colitis was exacerbated. Antiviral therapy improved his symptoms without augmenting the immunosuppressive therapy. CMV colitis should be considered when a patient with irColitis shows resistance to immunosuppressive therapy. 


\section{Case Reports in Gastroenterology}

Case Rep Gastroenterol 2020;14:103-109

DOI: $10.1159 / 000506186$

(c) 2020 The Author(s). Published by S. Karger AG, Basel www.karger.com/crg

Furuta et al.: Cytomegalovirus Enterocolitis in a Patient with Refractory Immune-Related Colitis

\section{Introduction}

Immune checkpoint inhibitors (ICIs), which bind to cytotoxic T-lymphocyte-associated protein 4 (CTLA-4) and programmed death receptor 1 (PD-1) on T cells or its ligand PD-L1, have improved the survival of patients with various types of advanced cancer [1-3]. Although ICIs can have remarkable responses, their use can be accompanied by unique immune-related adverse events (irAEs). The colon is one of the most frequently affected organs by irAEs, and immune-related colitis (irColitis) can be a life-threatening event, with colon perforation in some cases [4].

Immunosuppressive drugs, such as corticosteroids and infliximab, had been used to treat irAEs according to established treatment algorithms. In cases of irColitis that do not respond to corticosteroids, increasing the corticosteroid dose or switching to infliximab is recommended [5]. In most cases, irColitis can be resolved by appropriate treatment with corticosteroids, with or without infliximab. However, these treatments may increase the susceptibility to infections. Recently, a few reports showed that cytomegalovirus (CMV) reactivation might be involved in the pathogenesis of refractory gastrointestinal irAEs [6, 7].

In this report we present a case of unresectable malignant melanoma who developed irColitis secondary to ipilimumab. Although the irColitis initially responded to corticosteroids, CMV reactivation occurred and exacerbated the colitis. Antiviral therapy for CMV colitis ameliorated the symptoms independently of immunosuppressive therapy.

\section{Case Presentation}

A 77-year-old man with unresectable malignant melanoma in the left nasal cavity was referred to our hospital. He had angina pectoris, hypertension, and lacunar infarction. He had no particular family history, and laboratory tests revealed no remarkable abnormality. He was administered nivolumab ( $3 \mathrm{mg} / \mathrm{kg}$ every 2 weeks) as first-line therapy. After the 4 th infusion of nivolumab he developed polymyalgia rheumatica, which we considered an irAE. He was treated with prednisolone at a maintenance dose of $15 \mathrm{mg} /$ day because exacerbation immediately occurred when tapering of prednisolone was attempted. After the 5th infusion of nivolumab, the nasal tumor increased in size, which was considered to be progressive disease.

Ipilimumab ( $3 \mathrm{mg} / \mathrm{kg}$ every 3 weeks) was administered as second-line therapy. After the 3rd infusion of ipilimumab he suffered from severe fatigue and paralytic ileus, which were attributed to ipilimumab, and was admitted to a local hospital. For this reason, ipilimumab therapy was discontinued; 4 weeks after the last infusion of ipilimumab, he developed grade 2 diarrhea that persisted for 10 days (Fig. 1).

Computed tomography revealed wall thickening of the sigmoid colon and rectum. Sigmoidoscopy confirmed signs of inflammation, with coarse mucosa that was easily bleeding and contained purulence and erosions; these endoscopic findings resembled ulcerative colitis (Fig. 2a). Infectious workup, including CMV antigenemia and Clostridium difficile in the stool, were negative. Based on these findings, he was diagnosed with irColitis. Two weeks after the onset of diarrhea he was transferred to our hospital and was treated with daily intravenous $45 \mathrm{mg}$ of methylprednisolone $(1 \mathrm{mg} / \mathrm{kg}$ ), which led to a rapid reduction in the frequency of diarrhea.

On the 6th day after hospitalization, sigmoidoscopy showed mucosal healing in the rectum (Fig. 2b). On the 7th day after hospitalization, methylprednisolone was reduced to $35 \mathrm{mg} /$ day, but hematochezia and anemia were observed the following day. To confirm the 


\section{Case Reports in Gastroenterology}

Case Rep Gastroenterol 2020;14:103-109

DOI: $10.1159 / 000506186$

(c) 2020 The Author(s). Published by S. Karger AG, Basel www.karger.com/crg

Furuta et al.: Cytomegalovirus Enterocolitis in a Patient with Refractory Immune-Related Colitis

source of bleeding, left-sided colonoscopy was performed on the 12th day after hospitalization and revealed multiple punched-out ulcers in the descending colon, but the remission in the sigmoid colon and rectum was maintained (Fig. 2c). Histologic examination of the biopsy samples from the ulcers showed crypt abscesses and infiltration of lymphocytes and plasma cells (Fig. 2d). Immunohistochemical study was positive for CMV (Fig. 2e), although CMV pp65 antigenemia was negative. He was diagnosed with CMV colitis, for which ganciclovir $(5 \mathrm{mg} / \mathrm{kg}$ ) twice daily was initiated. Methylprednisolone was tapered to $30 \mathrm{mg} /$ day.

On the 27th day after hospitalization, total colonoscopy showed ulcers with healing tendency in the ascending and descending colon. However, the hematochezia and anemia did not improve. To rule out bleeding from the small intestine, video capsule endoscopy was performed on the 33rd day after hospitalization and revealed active bleeding from the ileum. On the 36th day after hospitalization, double balloon enteroscopy showed ulcers with healing tendency in the ileum (Fig. 2f). Although CMV was not detected from the biopsy samples of ileal ulcers at this time, this healing tendency could be due to the antiviral therapy. Thereafter, there was no recurrence of the hematochezia, and the anemia improved gradually. On the 40th day after hospitalization, ganciclovir was discontinued and steroids were tapered gradually. He was discharged on the 69th day after hospitalization.

\section{Discussion}

We herein report a case of CMV enterocolitis under corticosteroid therapy for irColitis. In irColitis with corticosteroid-refractory behavior, total colonoscopy and histologic examination should be conducted to rule out CMV reactivation. In this case, a biopsy of colon ulcer provided the definite diagnosis of CMV enterocolitis, and antiviral therapy improved enterocolitis without augmenting immunosuppressive therapy.

Cancer immunotherapy with ICIs has revolutionized the treatment of various types of advanced cancer [1-3]. Although ICIs can afford clinical benefits, they may cause several side effects called irAEs [4] that involve any organs, including the colon. irColitis causes diarrhea, bloody stool, abdominal pain, high fever, and, in some cases, colon perforation that can be a life-threatening event.

The incidence of diarrhea had been reported to be 12.1-13.7\% for anti-PD-1 and 30.2$35.4 \%$ for anti-CTLA-4, whereas the incidence of colitis had been reported to be $0.7-1.6 \%$ for anti-PD-1, 5.7-9.1\% for anti-CTLA-4, and $13.6 \%$ for the combination of both agents [8]. The endoscopic findings of irColitis closely resemble those of ulcerative colitis and include erythema, loss of vascular pattern, erosions, ulcerations, and luminal bleeding [4]. In most cases the sigmoid colon and rectum are involved, and therefore sigmoidoscopy had been usually sufficient to make a diagnosis of irColitis, and total colonoscopy can be avoided to decrease the risk of perforation [9]. According to this evidence, we performed sigmoidoscopy at the onset of diarrhea, and endoscopic findings led to the diagnosis of irColitis. Histologically, acute colitis can present as mononuclear cell and neutrophil infiltration of the lamina propria [9]. Blood examination and stool analysis should be carried out to rule out enteropathogens such as CMV and C. difficile [5, 8].

For the treatment of irColitis, corticosteroids have been used according to established treatment algorithms [5]. In corticosteroid-refractory or recurrent cases during the course of corticosteroid tapering, increasing the dose of corticosteroids or switching to infliximab has been recommended. In most cases, irColitis can be resolved by appropriate treatment with 


\section{Case Reports in Gastroenterology}

Case Rep Gastroenterol 2020;14:103-109

DOI: $10.1159 / 000506186$

c) 2020 The Author(s). Published by S. Karger AG, Basel www.karger.com/crg

Furuta et al.: Cytomegalovirus Enterocolitis in a Patient with Refractory Immune-Related Colitis

corticosteroids, with or without infliximab. However, corticosteroids increase the risk of infections, including CMV reactivation [10].

CMV is a double-stranded DNA virus that belongs to the herpesvirus family. Primary CMV infection can usually become an asymptomatic persistent infection and reactivate when patients become immunocompromised. Latent CMV infection is confirmed by positivity for CMVspecific IgG. CMV seroprevalence generally increases with age and varies by race and ethnicity $[11,12]$. Although we did not check the CMV serostatus, CMV reactivation was strongly suspected in this case because nearly $100 \%$ of blood donors aged $>60$ years were positive for CMV-specific IgG in Japan [13].

CMV colitis commonly occurs in immunocompromised hosts, such as patients under corticosteroid therapy. Recently, two reports showed CMV reactivation in patients with refractory irColitis during immunosuppressive therapy. Lankes et al. [6] reported a first case of irColitis that was resistant to corticosteroids and infliximab due to CMV reactivation. Franklin et al. [7] reported that 5 of 41 patients with irColitis were refractory to immunosuppressive therapy, and all these therapy-refractory cases had detectable CMV. In this report, we showed the first Japanese irColitis case that presented with recurrent colitis caused by CMV reactivation during the steroid tapering period. Although the mechanism of CMV reactivation during ICI therapy was not clarified, the immunosuppressive treatments for irAE likely induced CMV reactivation. Indeed, all reported cases with CMV reactivation during ICI treatment were under immunosuppressive treatments, such as corticosteroids or infliximab. In the present case, long-term administration of prednisolone for polymyalgia rheumatica and additional methylprednisolone for irColitis might have caused CMV reactivation.

CMV colitis and gastrointestinal irAE present with similar symptoms, such as diarrhea, bloody stool, abdominal pain, high fever, and ulceration in the intestine. Therefore, exclusion of CMV colitis is important before augmenting immunosuppressive therapy in refractory gastrointestinal irAE patients. Punched-out ulceration, which was shown in the present case, is one of the most common endoscopic characteristics of CMV colitis [14]. However, it has been unclear whether this punched-out ulceration would suggest CMV reactivation during the treatment of irColitis, because evidence on its endoscopic characteristics is scarce.

Since CMV antigenemia in the blood is often negative, immunohistochemistry or polymerase chain reaction of tissue samples is needed for an accurate diagnosis of CMV enterocolitis [15]. In the present case, CMV antigenemia was negative, and the diagnosis of CMV enterocolitis was made based on the multiple colonic and small intestinal ulcers detected on total colonoscopy, video capsule endoscopy, and double balloon enteroscopy. Therefore, evaluation of the entire colon, and the small intestine in some cases, would be required to diagnose steroid-refractory irColitis. In this case, the definitive diagnosis of CMV enterocolitis was confirmed by histologic examination of the punched-out ulcers in the colon and by the resolution of hematochezia with antiviral therapy, without the need to increase corticosteroids or add immunotherapy.

In conclusion, since CMV reactivation can occur during the treatment of gastrointestinal irAE, patients who exhibit resistance or recurrence with corticosteroids may need evaluation of the entire colon, and sometimes the small intestine, in order to rule out CMV reactivation before escalating immunosuppressive therapy.

\section{Acknowledgement}

The authors thank Kumiko I for secretarial assistance. 
Furuta et al.: Cytomegalovirus Enterocolitis in a Patient with Refractory Immune-Related Colitis

\author{
Statement of Ethics
}

Consent was obtained from the patient.

\section{Disclosure Statement}

The authors declare no financial disclosures or conflicts of interest.

\section{Funding Sources}

There were no funding sources.

\section{Author Contributions}

Y. Furuta, H. Miyamoto, M. Shimoda, Y. Hinokuma, T. Miyamura, A. Miyashita, and S. Fukushima carried out and confirmed the diagnosis, provided the details of the case, and contributed to the design of the report. Y. Furuta, H. Miyamoto, H. Naoe, M. Tanaka, and Y. Sasaki drafted the manuscript. All authors read and approved the final version of the manuscript.

\section{References}

1 Hodi FS, O'Day SJ, McDermott DF, Weber RW, Sosman JA, Haanen JB, et al. Improved survival with ipilimumab in patients with metastatic melanoma. N Engl J Med. 2010 Aug;363(8):711-23.

2 Kang YK, Boku N, Satoh T, Ryu MH, Chao Y, Kato K, et al. Nivolumab in patients with advanced gastric or gastro-oesophageal junction cancer refractory to, or intolerant of, at least two previous chemotherapy regimens (ONO-4538-12, ATTRACTION-2): a randomised, double-blind, placebo-controlled, phase 3 trial. Lancet. 2017 Dec;390(10111):2461-71.

3 Brahmer J, Reckamp KL, Baas P, Crinò L, Eberhardt WE, Poddubskaya E, et al. Nivolumab versus Docetaxel in Advanced Squamous-Cell Non-Small-Cell Lung Cancer. N Engl J Med. 2015 Jul;373(2):123-35.

4 Geukes Foppen MH, Rozeman EA, van Wilpe S, Postma C, Snaebjornsson P, van Thienen JV, et al. Immune checkpoint inhibition-related colitis: symptoms, endoscopic features, histology and response to management. ESMO Open. 2018 Jan;3(1):e000278.

5 Haanen JBAG, Carbonnel F, Robert C, Kerr KM, Peters S, Larkin J, et al. Management of toxicities from immunotherapy: ESMO Clinical Practice Guidelines for diagnosis, treatment and follow-up. Ann Oncol. 2017 Jul;28(suppl 4):iv119-42.

6 Lankes K, Hundorfean G, Harrer T, Pommer AJ, Agaimy A, Angelovska I, et al. Anti-TNF-refractory colitis after checkpoint inhibitor therapy: possible role of CMV-mediated immunopathogenesis. Oncoimmunology. 2016 Feb;5(6):e1128611.

7 Franklin C, Rooms I, Fiedler M, Reis H, Milsch L, Herz S, et al. Cytomegalovirus reactivation in patients with refractory checkpoint inhibitor-induced colitis. Eur J Cancer. 2017 Nov;86:248-56.

8 Soularue E, Lepage P, Colombel JF, Coutzac C, Faleck D, Marthey L, et al. Enterocolitis due to immune checkpoint inhibitors: a systematic review. Gut. 2018 Nov;67(11):2056-67.

9 Marthey L, Mateus C, Mussini C, Nachury M, Nancey S, Grange F, et al. Cancer Immunotherapy with AntiCTLA-4 Monoclonal Antibodies Induces an Inflammatory Bowel Disease. J Crohns Colitis. 2016 Apr; 10(4): 395-401.

10 Ko JH, Peck KR, Lee WJ, Lee JY, Cho SY, Ha YE, et al. Clinical presentation and risk factors for cytomegalovirus colitis in immunocompetent adult patients. Clin Infect Dis. 2015 Mar;60(6):e20-6.

11 Nakase H, Herfarth H. Cytomegalovirus Colitis, Cytomegalovirus Hepatitis and Systemic Cytomegalovirus Infection: Common Features and Differences. Inflamm Intest Dis. 2016 Apr;1(1):15-23.

12 Cannon MJ, Schmid DS, Hyde TB. Review of cytomegalovirus seroprevalence and demographic characteristics associated with infection. Rev Med Virol. 2010 Jul;20(4):202-13. 
Furuta et al.: Cytomegalovirus Enterocolitis in a Patient with Refractory Immune-Related Colitis

13 Furui Y, Satake M, Hoshi Y, Uchida S, Suzuki K, Tadokoro K. Cytomegalovirus (CMV) seroprevalence in Japanese blood donors and high detection frequency of CMV DNA in elderly donors. Transfusion. 2013 Oct;53(10):2190-7.

14 Hirayama Y, Ando T, Hirooka Y, Watanabe O, Miyahara R, Nakamura M, et al. Characteristic endoscopic findings and risk factors for cytomegalovirus-associated colitis in patients with active ulcerative colitis. World J Gastrointest Endosc. 2016 Mar;8(6):301-9.

15 Nakase H, Honzawa Y, Toyonaga T, Yamada S, Minami N, Yoshino T, et al. Diagnosis and treatment of ulcerative colitis with cytomegalovirus infection: importance of controlling mucosal inflammation to prevent cytomegalovirus reactivation. Intest Res. 2014 Jan;12(1):5-11.

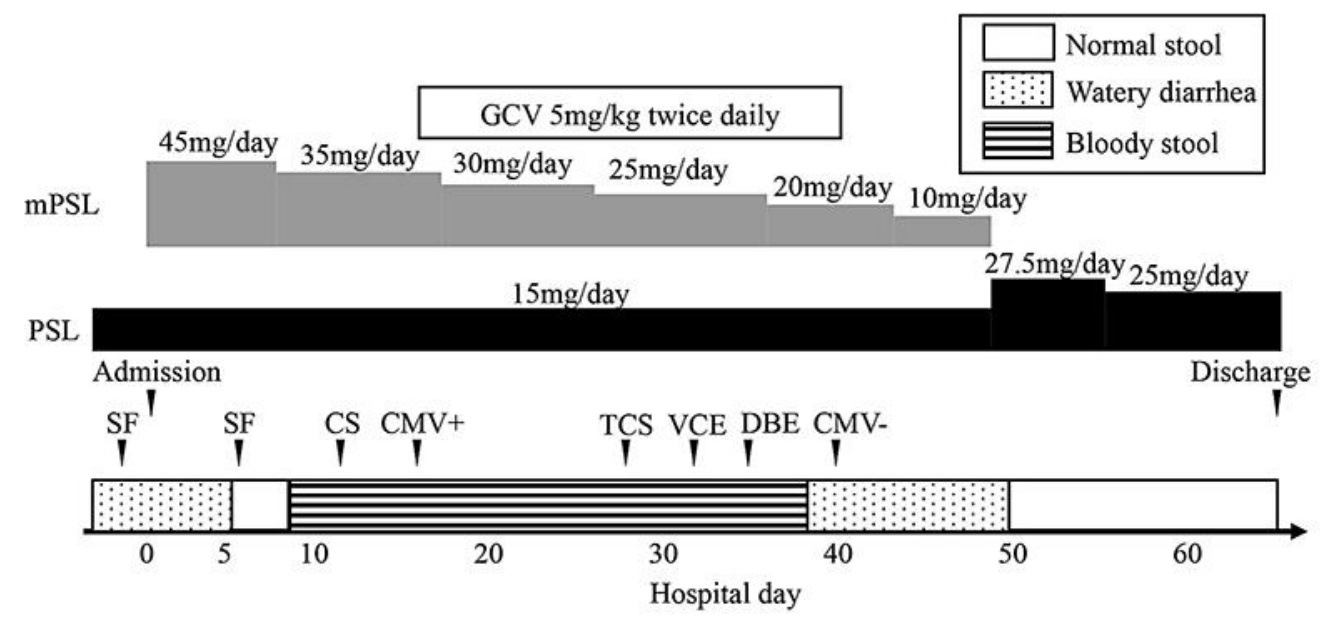

Fig. 1. The clinical course of the patient. The bar on the bottom indicates the stool quality. mPSL, methylprednisolone; PSL, prednisolone; GCV, ganciclovir; CMV, cytomegalovirus; SF, sigmoidoscopy; CS, colonoscopy; TCS, total colonoscopy; VCE, video capsule endoscopy; DBE, double balloon enteroscopy. 


\section{Case Reports in Gastroenterology}

\begin{tabular}{l|l}
\hline Case Rep Gastroenterol 2020;14:103-109 \\
\hline DOI: 10.1159/000506186 & $\begin{array}{l}\text { @ 2020 The Author(s). Published by S. Karger AG, Basel } \\
\text { www.karger.com/crg }\end{array}$ \\
\hline
\end{tabular}

Furuta et al.: Cytomegalovirus Enterocolitis in a Patient with Refractory Immune-Related Colitis
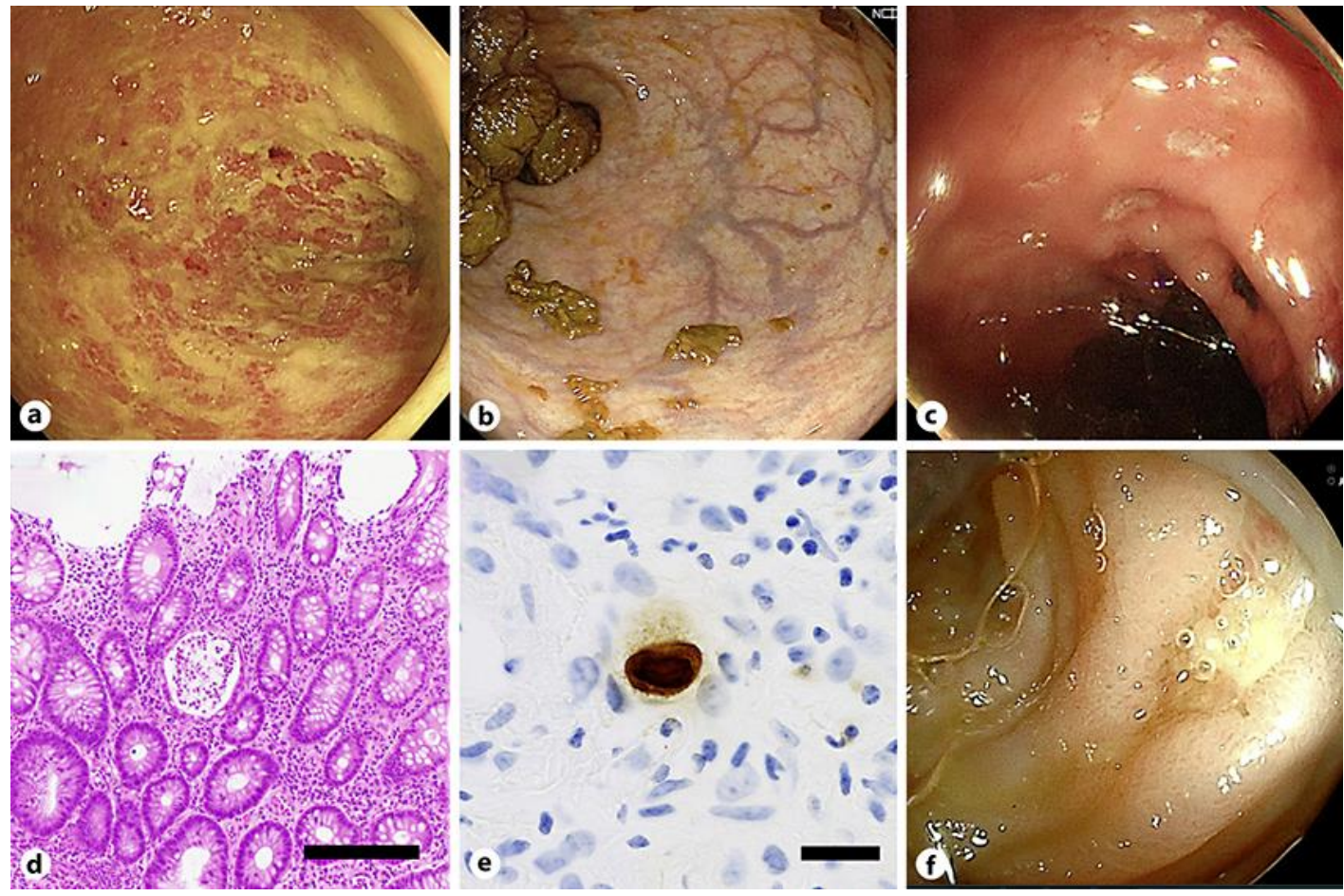

Fig. 2. Endoscopic and histologic findings. a Sigmoidoscopy before methylprednisolone therapy showed easy bleeding and coarse mucosa in the rectum. b Sigmoidoscopy on the 6th hospitalization day demonstrated almost normal rectal mucosa. c Colonoscopy on the 12th day showed multiple punched-out ulcers in the descending colon. $\mathbf{d}$ Histologic examination of the ulcers showed crypt abscess and inflammatory cell infiltration (hematoxylin and eosin staining). Original magnification, $\times 4$. Scale bar, $200 \mu \mathrm{m}$. e Immunohistochemistry for cytomegalovirus was positive. Original magnification, $\times 40$. Scale bar, $20 \mu \mathrm{m}$. f Double balloon enteroscopy demonstrated multiple ulcers in the small intestine. 